\title{
Thyroid dysfunction and risk factors in patients with newly diagnosed type 2 diabetes: A cross-sectional study
}

\section{Yanli Li}

Guangzhou Medical University https://orcid.org/0000-0002-9212-1752

Min Yi

Guangzhou Medical University

\section{Xiaoyi Deng}

Guangzhou Medical University

\section{Singla Sethiel Mosha}

Guangzhou Medical University

Wangen Li

Guangzhou Medical University

Xiaodan Zhang ( $\square$ sysuzhangxd@163.com )

https://orcid.org/0000-0001-8621-1113

\section{Research article}

Keywords: Type 2 diabetes mellitus; Thyroid dysfunction; Thyroid hormones

Posted Date: November 22nd, 2019

DOI: https://doi.org/10.21203/rs.2.16807/v2

License: (9) (i) This work is licensed under a Creative Commons Attribution 4.0 International License.

Read Full License 


\section{Abstract}

Background : Diabetes mellitus (DM) and thyroid dysfunction (TD) are two closely associated disorders. The coexistence of TD could adversely influence metabolic control and even increase the long-term mortality in patients with DM. The objective of the present study was to investigate the prevalence and risk factors of TD in patients with type 2 DM (T2DM). Methods : This is an observational cross-sectional study. A total of 340 patients with newly diagnosed T2DM who were admitted to ward of endocrinology department were included for analysis. Thyroid function was examined and its relationship with demographic, metabolic and diabetes-related parameters were evaluated Results : The prevalence of TD was $21.2 \%$ in the total population. The low T3 syndrome was the most frequent TD, in $14.7 \%$ of patients. Low FT3 level was associated with diabetic complications including presence of diabetic ketosis (DK) or diabetic ketoacidosis (DKA) $(r=-0.388, P \leq 0.001)$ and microalbuminuria $(r=-0.302, P \leq 0.001)$. Metabolic and demographic factors, including age, glycemic control and insulin resistance also correlated with levels of thyroid hormones. DK or DKA (OR $=6.161, \mathrm{P} \leq 0.001)$ and microalbuminuria (OR $=3.950, P=0.002$ ) were risk factors of low T3 syndrome. Conclusion : TD is not rarely seen in patients with newly diagnosed T2DM. Diabetic complications and diabetes-related metabolic and demographic factors are related to the presence of TD.

\section{Background}

Diabetes mellitus (DM) and thyroid dysfunction (TD) are two closely associated disorders. TD is more common in patients with type 2 DM (T2DM) than in those without diabetes and can adversely influence metabolic control $[1,2]$. In the long-term, the onset of TD can further increase the morbidity and mortality associated with diabetes [2]. The relationship between T2DM and TD is complex and the underlying mechanisms have not been fully elucidated. Several studies have investigated the prevalence and risk factors of TD in patients with T2DM. But researches in newly diagnosed patients were relatively rare. The objective of this study was to investigate the prevalence of TD and determine the risk factors in patients with newly diagnosed T2DM.

\section{Methods}

\section{Study population and design}

We studied patients with newly diagnosed T2DM who were admitted to ward of the Department of Endocrinology, The Second Affiliated Hospital of Guangzhou Medical University, from January 2014 to June 2019. The inclusion criteria were adults with newly diagnosed and treated T2DM based on the diagnostic criteria recommended by the Chinese Diabetes Society [3]. The exclusion criteria included: A. with history of thyroid disease or thyroid surgery; B. severe primary liver and kidney dysfunctions; C. using drugs potentially altering thyroid hormone concentrations such as amiodarone, beta-blockers and corticosteroids. A total of 340 patients with newly diagnosed T2DM were finally enrolled. 


\section{Measurement and data collection}

Demographic information including family history and habit of smoking was collected through the review of medical records. Body mass index (BMI) was calculated as weight $(\mathrm{kg})$ divided by squared height $(\mathrm{m})$. Blood pressure (BP) was detected twice in a sitting position after a 10-minute rest period and recorded as a mean of the two successive measurements. Hypertension was defined as systolic BP $\geq 140 \mathrm{mmHg}$ and/or diastolic BP $\geq 90 \mathrm{mmHg}$, or with positive histories of hypertension. Venous blood samples were collected in the morning after an overnight fast for laboratory measurement. Serum free triiodothyronine (FT3), free thyroxine (FT4) and thyroid stimulating hormone (TSH) were measured using electrochemiluminescence immunoassays. Normal ranges were as follows: TSH 0.4-5.0 mIU/L, FT3 2.63$5.70 \mathrm{pmol} / \mathrm{L}$, and FT4 9.01-19.05 pmol/L. Euthyroid was considered if thyroid hormone levels fall within reference range and thyroid dysfunction was considered if thyroid hormones fall outside the reference range. Subclinical hypothyroidism was defined as a serum TSH of more than $5.0 \mathrm{mIU} / \mathrm{L}$, in combination with a normal FT4. Overt hypothyroidism was defined as a serum TSH of more than $5.0 \mathrm{mIU} / \mathrm{L}$, in combination with a subnormal FT4. Subclinical hyperthyroidism was defined as a TSH of less than 0.4 $\mathrm{mIU} / \mathrm{L}$ with normal FT4. Overt hyperthyroidism was defined as a TSH of less than $0.4 \mathrm{mIU} / \mathrm{L}$ with elevated FT4. Low T3 syndrome was defined as a FT3 level of less than $2.63 \mathrm{pmol} / \mathrm{L}$ and/or a FT4 level of less than $9.01 \mathrm{pmol} / \mathrm{L}$, combined with a normal or subnormal TSH. Routine biochemical parameters (including fasting plasma glucose, 2-h postprandial plasma glucose, C-Peptide, glycated hemoglobin (HbA1c), serum high-density lipoprotein cholesterol (HDL-C), low-density lipoprotein cholesterol (LDL-C), total cholesterol, triglyceride, uric acid, serum creatinine) were measured by routine laboratory methods. The estimated glomerular filtration rate (eGFR) was calculated according to Modification of Diet in Renal Disease equation: eGFR $\left(\mathrm{mL} / \mathrm{min} / 1.73 \mathrm{~m}^{2}\right)=186 \times(\mathrm{SCr} / 88.4)^{-1.154} \times(\text { age })^{-0.203} \times(0.742$ if female $)$ [4] . Urine samples of 24 hours were collected to measure urine albumin levels using a chemiluminescence assay. Spot urinary samples of patients were collected at 7:00-8:00 am. Urinary albumin concentration was measured by nephelometry immunoassay and urinary creatinine concentration was measured by velocity method. The average value of the urinary albumin-to-creatinine ratio (UACR) was calculated. Microalbuminuria was defined as a UACR of $30-300 \mathrm{mg} / \mathrm{g}$ or an albumin excretion rate (AER) of 30-300 $\mathrm{mg} / 24$ hours. Homeostatic model assessment of insulin resistance (HOMA-IR) and $\beta$ cell function $($ HOMA- $\beta$ ) was calculated using well-established methods: $\mathrm{HOMA-IR}=1.5+$ fasting blood glucose $(\mathrm{mmol} / \mathrm{L}) \times$ fasting C-peptide $(\mathrm{pmol} / \mathrm{L}) / 2800, \mathrm{HOMA}-\beta=0.27 \times$ fasting C-peptide $(\mathrm{pmol} / \mathrm{L}) /($ fasting blood glucose $(\mathrm{mmol} / \mathrm{L})-3.5))[5]$

\section{Statistical analysis}

For continuous variables like thyroid hormones, student's t-test or one-way analysis of variance (ANOVA) were used. Chi-square test was used for categorical variables. Numeric values were presented as mean \pm 
standard deviation (SD) and categorical values were presented as number (\%). For evaluation of correlation between FT3, FT4, FT3/FT4 ratio or TSH and other variables, Pearson correlation coefficient was calculated. Factors associated with low T3 syndrome was analyzed with multivariate logistic regression. A two-sided $P$ value $<0.05$ was considered statistically significant. All statistical analyses were performed using IBM SPSS statistical software version 22 for Windows (IBM Corp., Armonk, New York, USA).

\section{Results}

\section{Baseline characteristics}

Detailed baseline demographic and clinical characteristics of included patients were presented in Table 1. The mean ( $\pm S D)$ age of the subjects was 54.85 ( \pm 14.1 ) years old, ranging from 18 to 90 years. Male patients constituted the majority $(n=221,65.0 \%)$. The mean $( \pm S D)$ BMI of the patients was $24.63( \pm$ $3.79) \mathrm{kg} / \mathrm{m}^{2}$. About $30 \%$ of the study population had a family history of diabetes. Social history of smoking was reported by $28.5 \%$ of the patients. Mean ( \pm SD) HbA1c level was $11.9( \pm 2.5) \%$. TD was found in 72 patients $(21.2 \%)$.

\section{Analyses of associated factors of thyroid function}

Among the categories of thyroid disorders, low T3 syndrome $(n=50,14.7 \%)$ was the most common form, followed by subclinical hyperthyroidism $(n=14,4.1 \%)$, hypothyroidism $(n=6,1.8 \%)$ and subclinical hypothyroidism $(n=2,0.6 \%)$. Higher prevalence of TD was found in patients over 60 years old $(n=32$, $27.4 \%)$ than in younger patients $(n=40,17.2 \%)(P=0.044)$. We found a lower level of FT3 $(3.21 \pm 0.74$ $\mathrm{pmol} / \mathrm{L}$ ) in patients with diabetic ketosis (DK) or diabetic ketoacidosis (DKA) than i) patients without DK

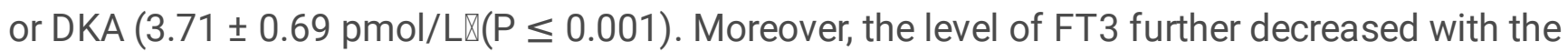
deterioration of DK $(2.60 \pm 1.23 \mathrm{pmol} / \mathrm{L})(\mathrm{P}=0.005)$. The level of FT4 $(12.54 \pm 3.64 \mathrm{pmol} / \mathrm{L})$ was also significantly lower in patients with DKA $(P=0.004)$. But no difference was shown between patients with DK $(14.75 \pm 2.80 \mathrm{pmol} / \mathrm{L})$ and patients without DK $(14.63 \pm 2.63 \mathrm{pmol} / \mathrm{L})$ in FT4 level. Lower level of FT3 $(3.12 \pm 0.86 \mathrm{pmol} / \mathrm{L})$ was found in patients with microalbuminuria as well $(P \leq 0.001)$. Levels of FT4 and TSH were also lower in patients with microalbuminuria than in patients with normoalbuminuria. But no significant difference was shown. The levels of FT3 and FT4 were lower in patients over 60 years old $(\mathrm{FT3} 3.32 \pm 0.77 \mathrm{pmol} / \mathrm{L}, \mathrm{FT} 414.08 \pm 2.80 \mathrm{pmol} / \mathrm{L})$ than in patients with younger age $(\mathrm{FT} 33.64 \pm 0.81$ $\mathrm{pmol} / \mathrm{L}, \mathrm{FT} 414.78 \pm 2.74 \mathrm{pmol} / \mathrm{L})(\mathrm{P} \leq 0.001, \mathrm{P}=0.027$, respectively). Pearson Correlation analysis revealed negative factors of FT3 level including DK or DKA, microalbuminuria, age and $\mathrm{HbA} 1 \mathrm{c}$. Positive correlated factors of FT3 level included eGFR, diastolic blood pressure (DBP), fasting C-peptide, 2-h Cpeptide, fasting insulin, 2-h insulin and HOMA-IR. DK or DKA and age remained to be negative correlated factors of FT4. DK or DKA, microalbuminuria and $\mathrm{HbA} 1 \mathrm{c}$ remained to be negative correlated factors of 
FT3/FT4 ratio. None of the metabolic or demographic parameters was strongly associated with TSH level (Table 2).

\section{Analyses of associated factors of low T3 syndrome}

Comparisons between patients with low T3 syndrome and patients with normal thyroid function was shown in Table 1. The presence of low T3 syndrome was significantly associated with DK or DKA, microalbuminuria, HbA1c, fasting C-Peptide, 2-h C-Peptide, 2-h insulin, HOMA-IR and DBP. Multivariate logistic regression analysis revealed that $\mathrm{DK}$ or DKA (OR $=6.161, P \leq 0.001)$, microalbuminuria (OR = 3.950, $P=0.002)$, HOMA-IR $(\mathrm{OR}=0.952, P=0.005)$ and eGFR level $(\mathrm{OR}=0.982, P=0.026)$ were associated factors of low T3 syndrome, among which DK or DKA and microalbuminuria were risk factors (Table 3).

\section{Discussion}

The prevalence of TD varied in patients with DM in different regions, ranging from $4 \%$ to over $20 \%$ [2]. The differences can be explained by the large population diversity, the varied degree of iodine intake, different diagnostic criteria of TD and different sensitivities of laboratory assays [6]. Subclinical hypothyroidism or hypothyroidism was reported to be the most common form of TD in several studies [6-9]. In this study, a relatively high prevalence of TD was found, among which low T3 syndrome constituted the majority.

Low T3 syndrome, also known as euthyroid sick syndrome (ESS) or nonthyroidal illness syndrome (NTIS), was initially described in the 1970s. It represents a state of alterations in thyroid hormone economy, which usually present in critically ill patients [10]. The low T3 syndrome is characterized by decreased serum T3 and T4 concentrations, increased serum reverse T3 (rT3) concentrations and unaltered or inappropriately low serum TSH [11]. Complicated mechanisms were involved in its pathogenesis, including downregulation of TRH and TSH production, changes in thyroid hormone metabolism and inhibitory effect of cytokines on the thyroid gland [12]. The presence of low T3 syndrome is a predictor of poor prognosis of acute or chronic illnesses.

DK or DKA was found to be a risk factor of low T3 syndrome in this study. Previous studies mainly focused on type 1 diabetes mellitus (T1DM), but showed similar results. TD including low T3 syndrome and hypothyroidism was more common in patients with DKA [13-16]. The presence of low T3 syndrome was associated with poor glycemic control $[13,14]$ and free thyroid hormones were correlated with the severity of DKA [14], which was in accordance with our findings. The decreased thyroid hormones could increase to normal soon after correction of DKA $[13,15]$.

The relationship between thyroid hormones and diabetic nephropathy (DN) is becoming a concern these years. A study in euthyroid subjects with T2DM showed that low levels of thyroid hormones (FT3 and FT4) were associated with DN [17]. DN was a risk factor of TD in patients with T2DM [8.9]. High levels of 
TSH and low levels of FT3 were observed in T2DM patients with DN [18]. Moreover, high levels of TSH and/or low levels of FT3 were associated with more severe proteinuria, renal insufficiency and glomerular lesions in patients with DN [19]. We also observed lower FT3 level and FT3/FT4 ratio in patients with microalbuminuria. The presence of low T3 syndrome was significantly associated with microalbuminuria. Recently, a study in adult euthyroid patients with T1DM showed that higher FT3 level was related to lower prevalence of microangiopathy and better metabolic control [20], which further supported our findings.

Levels of thyroid hormones were also suggested to be associated with some metabolic and demographic parameters. A study in non-diabetic individuals demonstrated that low T3 levels were significantly associated with decreased HOMA-IR, which indicated an association of thyroid function with insulin resistance [21]. FT3 and FT4 positively and negatively correlated with HOMA-IR and atherogenic lipid profiles, respectively, in a euthyroid population with obesity [22]. In euthyroid subjects, serum FT4 was negatively associated with and TSH was positively associated with insulin resistance. Also, FT4 was associated with risk of metabolic syndrome [23]. TSH and thyroid hormones were found to correlated with multiple cardiometabolic risk factors, with age- and sex-independent effects on cholesterol and glucose metabolism [24]. We also found some relationships between thyroid hormones and metabolic parameters including HOMA-IR, HbA1c, serum insulin and C-peptide levels. In some studies, obesity was also a risk factor of TD [8. 9]. Both FT3 and FT4 levels were positively correlated with BMI in euthyroid subjects with obesity [23]. Higher FT3 concentration correlated positively with markers of obesity such as BMI in euthyroid T1DM patients [20]. However, we did not find significant difference in levels of thyroid hormones between patients with obesity $\left(\mathrm{BMI} \geq 28 \mathrm{~kg} / \mathrm{m}^{2}\right)$ and patients with relatively normal BMI values. A recent large population-based study demonstrated that elevated TSH level within the normal range was a risk marker associated with a series of cardiometabolic changes including central obesity, insulin resistance, elevated BP, dyslipidemia, hyperuricemia, inflammation and hypercoagulability [25]. But in this study, we did not find significant relationship between TSH and other metabolic parameters.

Advanced age, long duration of diabetes and poor glycemic control were commonly indicated to be risk factors of low levels of FT3 and presence of TD in patients with T2DM [8. 9]. And the abnormalities seemed to be reversed upon restoration of metabolic control [26]. We also discovered higher prevalence of TD or low T3 syndrome in patients over 60 years old and patients with higher glycemic levels. TD was reported to be more common in female as compared to male patients with T2DM in many studies [9, 27. 28]. However, no gender difference was indicated in our study. This may be partly attributed to the different inclusion criteria. Most studies did not include low T3 syndrome as a form of TD. This may also explain the relatively higher prevalence of TD $(21.2 \%)$ in our study since low T3 syndrome contributed over $50 \%$ of the disorders. Furthermore, subjects in the present study were admitted in ward for treatment of diabetes. The conditions of patients, particularly glycemic control, were generally worse than the ones in outpatient clinics. Actually, most subjects in our study had a HbA1c level over $10 \%$. This may also contribute to the high prevalence of TD. 
There are several limitations of the present study. First, only limited number of patients in a single center were involved. The samples were derived from an inpatient setting and may not be representative of the true population. Second, due to the cross-sectional nature of this study, definite cause-and-effect relationships between TD and other abnormalities or factors could not be established. Therefore, our results should be interpreted with caution.

\section{Conclusions}

TD was not rare in patients with newly diagnosed T2DM. Low T3 syndrome was the most common subtype. Low FT3 level was associated with diabetic complications including presence of DK or DKA and microalbuminuria. Metabolic and demographic factors, including age, glycemic control and insulin resistance also correlated with levels of thyroid hormones. In the future, large prospective studies are needed to further investigate the prevalence of TD and to determine the association between TD and other factors in patients with T2DM.

\section{Abbreviations}

AER: Albumin excretion rate; ANOVA: one-way analysis of variance; BMI: Body mass index; BP: Blood pressure; DK: Diabetic ketosis; DKA: Diabetic ketoacidosis; DM: Diabetes mellitus; DN: Diabetic nephropathy; eGFR: Estimated glomerular filtration rate; FPG: Free plasma glucose; FT3: Free triiodothyronine; FT4: Free thyroxine; HbA1c: Glycated hemoglobin; HDL-C: High-density lipoprotein cholesterol; HOMA- $\beta$ : Homeostasis model assessment of $\beta$ cell function; HOMA-IR: Homeostasis model assessment of insulin resistance; LDL-C: Low-density lipoprotein cholesterol; PG: Plasma glucose; SD: Standard deviation; T2DM: Type 2 diabetes mellitus; T3: Triiodothyronine; TD: Thyroid dysfunction; TC: Total cholesterol; TG, Triglyceride; TSH: Thyroid stimulating hormone; UACR, Urinary albumin-to-creatinine ratio

\section{Declarations}

\section{Ethics approval and consent to participate}

This study was approved by the Ethics Committee of the Second Affiliated Hospital of Guangzhou University, Guangzhou, China. All procedures performed in the study were in accordance with the approved ethical standards and regulations. Informed consent was waived by the local ethics committee due to the retrospective nature of the study with no impact on health outcome.

\section{Consent to publish}

Not applicable.

\section{Availability of data and material}


The data used for analysis are available from the corresponding author on reasonable request.

\section{Competing interests}

All authors have no competing interests.

\section{Funding}

The present study was supported by grants from the National Natural Science Foundation of China (grant number 81800726), National Natural Science Foundation of China (grant no. 81800682), Natural Science Foundation of Guangdong, China (grant number 2017A030310257) and Science and Technology Program of Guangzhou, China (grant number 201707010045). The funders had no role in the study design, data analysis, interpretation and writing of the manuscript in this study.

\section{Authors' contributions}

$X Z$ designed the study. $X Z, Y L, Y M, D Y$, and SM collected the data. $X Z, Y L$, and $Y M$ analyzed the data. $X Z$ and $W L$ wrote the manuscript. All authors have read and approved the final manuscript.

\section{Acknowledgements}

None.

\section{Authors' Information}

Department of Endocrinology, The Second Affiliated Hospital of Guangzhou Medical University, 250 East Changgang Road, Haizhu District, Guangzhou 510260, China.

\section{References}

[1] Vondra K, Vrbikova J, Dvorakova K. Thyroid gland diseases in adult patients with diabetes mellitus. Minerva Endocrinol. 2005;30(4):217-36.

[2] Biondi B, Kahaly GJ, Robertson RP. Thyroid Dysfunction and Diabetes Mellitus: Two Closely Associated Disorders. Endocr Rev. 2019;40(3):789-824.

[3] Chinese Diabetes Society. Guideline for Type 2 Diabetes (2010). Beijing: Beijing University Medical Press; 2011.

[4] Levey AS, Bosch JP, Lewis JB, Greene T, Rogers N, Roth D. A more accurate method to estimate glomerular filtration rate from serum creatinine: a new prediction equation. Modification of Diet in Renal Disease Study Group. Ann Intern Med. 1999;130(6):461-70.

[5] Li X, Zhou ZG, Qi HY, Chen XY, Huang G. Replacement of insulin by fasting C-peptide in modified homeostasis model assessment to evaluate insulin resistance and islet beta cell function. Zhong Nan Da 
Xue Xue Bao Yi Xue Ban, 2004;29(4):419-23.

[6] Palma CC, Pavesi M, Nogueira VG, Clemente EL, Vasconcellos Mde F, Pereira LC Júnior, et al. Prevalence of thyroid dysfunction in patients with diabetes mellitus. Diabetol Metab Syndr. 2013;5(1):58.

[7] Jali MV, Kambar S, Jali SM, Pawar N, Nalawade P. Prevalence of thyroid dysfunction among type 2 diabetes mellitus patients. Diabetes Metab Syndr. 2017;11 Suppl 1:S105-8.

[8] Ogbonna SU, Ezeani IU. Risk Factors of Thyroid Dysfunction in Patients With Type 2 Diabetes Mellitus. Front Endocrinol (Lausanne). 2019;10:440.

[9] Ozair M, Noor S, Raghav A, Siddiqi SS, Chugtai AM, Ahmad J. Prevalence of thyroid disorders in North Indian Type 2 diabetic subjects: A cross sectional study. Diabetes Metab Syndr. 2018;12(3):301-4.

[10] Mebis L, Van den Berghe G. Thyroid axis function and dysfunction in critical illness. Best Pract Res Clin Endocrinol Metab. 2011;25(5):745-57.

[11] Docter R, Krenning EP, de Jong M, Hennemann G. The sick euthyroid syndrome: changes in thyroid hormone serum parameters and hormone metabolism. Clin Endocrinol (Oxf). 1993;39(5):499-518.

[12] de Vries EM, Fliers E, Boelen A. The molecular basis of the non-thyroidal illness syndrome. J Endocrinol. 2015;225(3):R67-81.

[13] Rashidi H, Ghaderian SB, Latifi SM, Hoseini F. Impact of diabetic ketoacidosis on thyroid function tests in type 1 diabetes mellitus patients. Diabetes Metab Syndr. 2017;11 Suppl 1:S57-S59.

[14] Hu YY, Li GM, Wang W. Euthyroid sick syndrome in children with diabetic ketoacidosis. Saudi Med J. 2015;36(2):243-7.

[15] Yang W, Sheng F. Factors associated with thyroid dysfunction in children with newly diagnosed type 1 diabetes mellitus. Minerva Pediatr. 2019 May 23.

[16] Fatourechi A, Ardakani HM, Sayarifard F, Sheikh M. Hypothyroidism among pediatric patients with type 1 diabetes mellitus, from patients' characteristics to disease severity. Clin Pediatr Endocrinol. 2017;26(2):73-80.

[17] Wang J, Li H, Tan M, Gao G, Zhang Y, Ding B, et al. Association between thyroid function and diabetic nephropathy in euthyroid subjects with type 2 diabetes mellitus: a cross-sectional study in China. Oncotarget. 2019;10(2):88-97.

[18] Zhao W, Li X, Liu X, Lu L, Gao Z. Thyroid function in patients with type 2 diabetes mellitus and diabetic nephropathy: A single center study. J Thyroid Res. 2018;2018:9507028. 
[19] Han Q, Zhang J, Wang Y, Li H, Zhang R, Guo R, et al. Thyroid hormones and diabetic nephropathy: An essential relationship to recognize. Nephrology (Carlton). 2019;24(2):160-9.

[20] Falkowski B, Rogowicz-Frontczak A, Grzelka A, Uruska A, Schlaffke J, Araszkiewicz A, et al. Higher free triiodothyronine concentration is associated with lower prevalence of microangiopathic complications and better metabolic control in adult euthyroid people with type 1 diabetes. Endocrine. 2018;60(3):458-65.

[21] Wang CY, Yu TY, Shih SR, Huang KC, Chang TC. Low total and free triiodothyronine levels are associated with insulin resistance in non-diabetic individuals. Sci Rep. 2018;8(1):10685.

[22] Temizkan S, Balaforlou B, Ozderya A, Avci M, Aydin K, Karaman S, et al. Effects of thyrotrophin, thyroid hormones and thyroid antibodies on metabolic parameters in a euthyroid population with obesity. Clin Endocrinol (Oxf). 2016;85(4):616-23.

[23] Mehran L, Amouzegar A, Tohidi M, Moayedi M, Azizi F. Serum free thyroxine concentration is associated with metabolic syndrome in euthyroid subjects. Thyroid. 2014;24(11):1566-74.

[24] Le TN, Celi FS, Wickham EP $3^{\text {rd }}$. Thyrotropin levels are associated with cardiometabolic risk factors in euthyroid adolescents. Thyroid. 2016;26(10):1441-9.

[25] Chang YC, Hua SC, Chang CH, Kao WY, Lee HL, Chuang LM, et al. High TSH levels within normal range is associated with obesity, dyslipidemia, hypertension, inflammation, hypercoagulability, and the metabolic syndrome: A novel cardiometabolic marker. J Clin Med. 2019;8(6). pii: E817.

[26] Moura Neto A, Zantut-Wittmann DE. Abnormalities of thyroid hormone metabolism during systemic illness: The low T3 syndrome in different clinical settings. Int J Endocrinol. 2016;2016:2157583.

[27] Papazafiropoulou A, Sotiropoulos A, Kokolaki A, Kardara M, Stamataki P, Pappas S. Prevalence of thyroid dysfunction among greek type 2 diabetic patients attending an outpatient clinic. J Clin Med Res. 2010;2(2):75-8.

[28] Zhu Y, Xu F, Shen J, Liu Y, Bi C, Liu J, et al. Prevalence of thyroid dysfunction in older Chinese patients with type 2 diabetes-A multicenter cross-sectional observational study across China. PLoS One. 2019;14(5):e0216151.

\section{Tables}

Table 1. Baseline and clinical characteristics of patients 


\begin{tabular}{|c|c|c|c|c|}
\hline Characteristics & Euthyroid & $\begin{array}{c}\text { Low T3 } \\
\text { syndrome }\end{array}$ & Total & $P$ \\
\hline \multicolumn{5}{|l|}{ Basic characteristics } \\
\hline $\mathrm{N}$ & 268 & 50 & 340 & \\
\hline $\begin{array}{l}\text { Age, year } \\
\geq 60 \\
<60\end{array}$ & $54.42(12.88)$ & $54.66(19.27)$ & $54.85(14.14)$ & 0.932 \\
\hline Gender & & & & 0.604 \\
\hline Male & $172(64.2)$ & $34(68.0)$ & $221(65.0)$ & \\
\hline Female & $96(35.8)$ & $16(32.0)$ & $119(35.0)$ & \\
\hline $\mathrm{BMI}, \mathrm{kg} / \mathrm{m}^{2}$ & $24.82(3.75)$ & $23.41(4.17)$ & $24.63(3.79)$ & $0.043^{*}$ \\
\hline Past history of hypertension & $94(35.1)$ & $17(34.0)$ & $124(36.5)$ & 0.884 \\
\hline Family history of diabetes & 79 (29.5) & $18(36.0)$ & $102(30.0)$ & 0.358 \\
\hline Smoking & $74(27.6)$ & $15(30.0)$ & $97(28.5)$ & 0.730 \\
\hline \multicolumn{5}{|l|}{ Thyroid function } \\
\hline FT3, pmol/L & $3.78(0.64)$ & $2.32(0.47)$ & $3.52(0.81)$ & $\begin{array}{c}\leq \\
0.001^{*}\end{array}$ \\
\hline FT4, pmol/L & $14.77(2.64)$ & $13.46(2.92)$ & $14.54(2.78)$ & $0.002^{*}$ \\
\hline $\mathrm{TSH}, \mu \mathrm{IU} / \mathrm{mL}$ & $1.61(1.00)$ & $1.04(0.68)$ & $1.66(1.80)$ & $\leq$ \\
\hline $\begin{array}{l}\text { Positive thyroid antibodies } \\
\text { Diabetes }\end{array}$ & $16(6.6)$ & $5(10.4)$ & $28(8.5)$ & $\begin{array}{l}0.001^{*} \\
0.444\end{array}$ \\
\hline $\mathrm{FPG}, \mathrm{mmol} / \mathrm{L}$ & $13.45(4.72)$ & $12.22(6.39)$ & $13.23(5.02)$ & 0.115 \\
\hline 2-h PG, $\mathrm{mmol} / \mathrm{L}$ & $20.41(7.47)$ & $18.93(5.86)$ & $20.27(7.28)$ & 0.432 \\
\hline $\mathrm{HbA1c}, \%$ & $11.7(2.5)$ & $12.6(2.4)$ & $11.9(2.5)$ & $0.011^{*}$ \\
\hline Fasting C-Peptide, $\mu \mathrm{g} / \mathrm{L}$ & $2.09(1.05)$ & $1.38(1.26)$ & $1.97(1.14)$ & $\begin{array}{l}\leq .01 \\
0.001^{*}\end{array}$ \\
\hline 2-h C-Peptide, $\mu \mathrm{g} / \mathrm{L}$ & $4.06(2.76)$ & $2.06(1.53)$ & $3.68(2.69)$ & $\begin{array}{c}\leq \\
0.001^{*}\end{array}$ \\
\hline Fasting insulin, mIU/L & $9.61(5.33)$ & $7.25(4.54)$ & $9.36(5.58)$ & 0.050 \\
\hline 2-h insulin, $\mathrm{mIU} / \mathrm{L}$ & $32.67(33.85)$ & $15.63(14.51)$ & $30.61(32.76)$ & $\begin{array}{c}\leq \\
0.001^{*}\end{array}$ \\
\hline HOMA- $\beta$ & $\begin{array}{l}239.61 \\
(298.74)\end{array}$ & $\begin{array}{c}231.66 \\
(264.02)\end{array}$ & $\begin{array}{l}235.90 \\
(286.28)\end{array}$ & 0.868 \\
\hline HOMA-IR & $31.51(19.35)$ & $20.81(19.97)$ & $29.88(20.72)$ & $0.001^{*}$ \\
\hline \multicolumn{5}{|l|}{ Complications } \\
\hline Acute complication & & & & $\begin{array}{c}\leq \\
0.001^{*}\end{array}$ \\
\hline Diabetic ketosis & $56(20.9)$ & $20(40.0)$ & $81(23.8)$ & \\
\hline Diabetic ketoacidosis & $4(1.5)$ & $14(28.0)$ & $20(5.9)$ & \\
\hline \multicolumn{5}{|l|}{ Chronic complication } \\
\hline Diabetic nephropathy & $50(18.7)$ & $26(52.0)$ & $81(23.8)$ & $\begin{array}{c}\leq \\
0.001^{*}\end{array}$ \\
\hline $\begin{array}{l}\text { Diabetic peripheral } \\
\text { neuropathy }\end{array}$ & $18(6.7)$ & $3(6.0)$ & $22(6.5)$ & $\begin{array}{l}0.001 \\
0.850\end{array}$ \\
\hline Diabetic retinopathy & $1(0.4)$ & $0(0.0)$ & $1(0.3)$ & 0.558 \\
\hline Diabetic foot ulcer & $1(0.4)$ & $0(0.0)$ & $1(0.3)$ & 0.558 \\
\hline Systolic BP, mm Hg & $134.3(18.9)$ & $131.4(21.8)$ & $134.0(19.4)$ & 0.336 \\
\hline Diastolic BP, mm Hg & $86.2(11.8)$ & $81.7(11.5)$ & $85.3(11.9)$ & $0.012^{*}$ \\
\hline HDL-C, $\mathrm{mmol} / \mathrm{L}$ & $1.44(6.43)$ & $0.89(0.36)$ & $1.33(5.71)$ & 0.548 \\
\hline LDL-C, mmol/L & $3.47(1.06)$ & $3.20(1.28)$ & $3.40(1.10)$ & 0.115 \\
\hline
\end{tabular}


Triglyceride, $\mathrm{mmol} / \mathrm{L}$

Total cholesterol, $\mathrm{mmol} / \mathrm{L}$

Uric acid, $\mu \mathrm{mol} / \mathrm{L}$

eGFR, $\mathrm{mL} / \mathrm{min} / 1.73 \mathrm{~m}^{2}$

Microalbuminuria
$2.50(2.59)$

$5.31(1.26)$

339.97

(217.5)

$91.98(23.13)$

50 (18.8)

$$
\begin{gathered}
3.80(7.99) \\
5.30(2.41) \\
360.04 \\
(160.13) \\
88.60(34.74) \\
26(53.1)
\end{gathered}
$$

$2.65(3.86)$

$5.26(1.49)$

343.79

(205.29)

$91.41(25.98)$

$81(23.8)$
0.259

0.992

0.535

0.511

$\leq$

$0.001^{*}$

BMI, body mass index; FT3, free triiodothyronine; FT4, free thyroxine; TSH, thyroid stimulating hormone; FPG, free plasma glucose; PG, plasma glucose; HbA1c, glycated hemoglobin; HOMA- $\beta$, homeostasis model assessment of $\beta$ cell function; HOMA-IR, homeostasis model assessment of insulin resistance; BP, blood pressure; HDL-C, highdensity lipoprotein cholesterol; LDL-C, low-density lipoprotein cholesterol; eGFR, estimated glomerular filtration rate; ${ }^{*} P$-vale $<0.05$.

Table 2 Correlation between thyroid function and demographic and metabolic parameters 


\begin{tabular}{|c|c|c|c|c|c|c|c|c|}
\hline \multirow[t]{2}{*}{ bles } & \multicolumn{2}{|c|}{ FT3 } & \multicolumn{2}{|c|}{ FT4 } & \multicolumn{2}{|c|}{ FT3/FT4 ratio } & \multicolumn{2}{|c|}{ TSH } \\
\hline & $\mathrm{r}$ & $P$ & $\mathrm{r}$ & $P$ & $\mathrm{r}$ & $P$ & $\mathrm{r}$ & $P$ \\
\hline & $\begin{array}{l}-0.168 \\
-0.16\end{array}$ & $0.002^{*}$ & -0.141 & $0.009^{*}$ & -0.101 & 0.063 & 0.063 & 0.247 \\
\hline & 0.079 & 0.159 & 0.002 & 0.971 & 0.073 & 0.195 & 0.080 & 0.156 \\
\hline & -0.388 & $\begin{array}{c}\leq \\
0.001^{*}\end{array}$ & -0.113 & $0.038^{*}$ & -0.348 & $\begin{array}{c}\leq \\
0.001^{*}\end{array}$ & 0.092 & 0.089 \\
\hline ıuria & -0.302 & $\begin{array}{c}\leq \\
0.001^{*}\end{array}$ & -0.072 & 0.187 & -0.272 & $\begin{array}{c}\leq \\
0.001^{*}\end{array}$ & -0.057 & 0.299 \\
\hline \multirow[t]{6}{*}{ ọheral } & 0.009 & 0.873 & 0.126 & $0.020^{*}$ & -0.068 & 0.212 & 0.031 & 0.564 \\
\hline & -0.224 & $\begin{array}{c}\leq \\
0.001^{*}\end{array}$ & 0.111 & $0.041^{*}$ & -0.273 & $\begin{array}{c}\leq \\
0.001^{*}\end{array}$ & 0.016 & 0.765 \\
\hline & 0.030 & 0.586 & 0.173 & $0.001^{*}$ & -0.042 & 0.442 & -0.019 & 0.733 \\
\hline & 0.065 & 0.230 & -0.001 & 0.985 & 0.113 & $0.039^{*}$ & -0.070 & 0.199 \\
\hline & 0.065 & 0.230 & 0.020 & 0.710 & 0.042 & 0.439 & -0.006 & 0.909 \\
\hline & -0.060 & 0.270 & -0.117 & $0.031^{*}$ & 0.033 & 0.547 & 0.012 & 0.827 \\
\hline arol & 0.002 & 0.968 & -0.067 & 0.218 & 0.090 & 0.097 & -0.075 & 0.169 \\
\hline tide & 0.241 & $\begin{array}{c}\leq \\
0.001^{*}\end{array}$ & 0.017 & 0.764 & 0.195 & $0.001^{*}$ & 0.065 & 0.262 \\
\hline : & 0.372 & $\begin{array}{c}\leq \\
0.001^{*}\end{array}$ & 0.025 & 0.670 & 0.347 & $\begin{array}{c}\leq \\
0.001^{*}\end{array}$ & 0.041 & 0.490 \\
\hline \multirow[t]{7}{*}{ in } & 0.173 & $0.020^{*}$ & -0.139 & 0.062 & 0.282 & $\begin{array}{c}\leq \\
0.001^{*}\end{array}$ & 0.010 & 0.892 \\
\hline & 0.167 & $0.020^{*}$ & -0.194 & $0.011^{*}$ & 0.337 & $\begin{array}{c}\leq \\
0.001^{*}\end{array}$ & -0.002 & 0.976 \\
\hline & 0.178 & $0.002^{*}$ & 0.070 & 0.225 & 0.112 & 0.053 & 0.105 & 0.071 \\
\hline & 0.042 & 0.470 & -0.080 & 0.168 & 0.051 & 0.381 & 0.071 & 0.905 \\
\hline & 0.150 & $0.006^{*}$ & 0.179 & $0.001^{*}$ & 0.044 & 0.417 & -0.046 & 0.396 \\
\hline & 0.043 & 0.434 & -0.067 & 0.216 & 0.035 & 0.521 & -0.045 & 0.413 \\
\hline & 0.176 & $0.001^{*}$ & 0.069 & 0.203 & 0.096 & 0.077 & -0.088 & 0.105 \\
\hline
\end{tabular}

FT3, free triiodothyronine; FT4, free thyroxine; TSH, thyroid stimulating hormone; BMI, body mass index; DK, diabetic ketosis; DKA, diabetic ketoacidosis; HbA1c, glycated hemoglobin; FPG, free plasma glucose; LDL-C, low-density lipoprotein cholesterol; HDL-C, high-density lipoprotein cholesterol; HOMA-IR, homeostasis model assessment of insulin resistance; HOMA- $\beta$, homeostasis model assessment of $\beta$ cell function; eGFR, estimated glomerular filtration rate; $\mathrm{BP}$, blood pressure; ${ }^{*} P$-vale $<0.05$.

Table 3 Multivariate analysis of associated factors of low T3 syndrome in patients with newly diagnosed T2DM 


\begin{tabular}{ccccc}
\hline Variable & OR & $\beta$ & Wald $\chi^{2}$ & $P$ \\
\hline DK or DKA & 6.161 & 1.818 & 26.733 & $\leq 0.001^{*}$ \\
Microalbuminuria & 3.950 & 1.376 & 9.365 & $0.002^{*}$ \\
\hline HOMA-IR & 0.952 & -0.049 & 7.946 & $0.005^{*}$ \\
eGFR & 0.982 & -0.018 & 4.969 & $0.026^{*}$ \\
\hline
\end{tabular}

T3, triiodothyronine; T2DM, type 2 diabetes mellitus; DK, diabetic ketosis; DKA, diabetic ketoacidosis; HOMA-IR, homeostasis model assessment of insulin resistance; eGFR, estimated glomerular filtration rate; ${ }^{*} P$-vale $<0.05$.

\section{Supplementary Files}

This is a list of supplementary files associated with this preprint. Click to download.

- STROBEchecklistcrosssectional.doc 